\title{
Community optometrist-led post-operative cataract care: how many patients re-present to the hospital ophthalmic department?
}

\author{
Amy O'Regan $^{1}$ (D) Aisling McGlacken-Byrne ${ }^{1} \cdot$ Shivona Chetty $^{1} \cdot$ Paul Mullaney $^{1}$
}

Received: 30 October 2020 / Accepted: 10 January 2021 / Published online: 25 January 2021

(C) Royal Academy of Medicine in Ireland 2021

\begin{abstract}
Background Cataract surgery represents a significant workload for ophthalmologists in Ireland. Post-operative shared care with community optometrists can reduce the need for hospital follow-up appointments.

Aims Eight years after the introduction of a shared-care pathway, we wished to quantify the proportion of patients discharged to the community for post-operative follow-up, and the number that re-present to the hospital due to cataract-related issues.

Methods We collected data on all patients who underwent cataract surgery in our centre over a three month period. Electronic patient records were used to establish whether the patient was discharged on the day of surgery, and whether they re-attended the department post-operatively. Post-operative complications were recorded.

Results 394 cataract procedures were carried out over the three months. 369 patients were discharged to an optometrist for their post-operative care. Of those, 38 were re-referred or re-presented to the hospital ophthalmic service. 21 of these had a postoperative complication. Complications included seven cases of post-operative uveitis, 5 patients with cystoid macular oedema, one retinal detachment and one retained lens fragment.

Conclusions Community optometrist-led post-operative care for uncomplicated patients is an effective way of reducing the workload associated with cataract surgery. Re-referral pathways must be in place to facilitate timely management of postoperative complications.
\end{abstract}

Keywords Cataract $\cdot$ Community $\cdot$ COVID-19 $\cdot$ Optometry $\cdot$ Shared care

\section{Introduction}

Cataract surgery is the most commonly performed day case surgical procedure in most European countries [1]. Over 12,000 cataract operations were carried out in public hospitals in Ireland in 2018 [2]. The number of people over 65 in Ireland is set to rise by $80 \%$ by 2036 [3], and demand for cataract procedures will follow a similar trend. A "lean" healthcare service facilitates appropriate resource allocation in order to meet demand [4]. Cutting down on unnecessary outpatient clinic visits is a step towards streamlining a highvolume service such as ophthalmology.

Amy O'Regan

amoregan@tcd.ie

1 Department of Ophthalmology, Sligo University Hospital, Sligo, Ireland
Cataract surgery carries a low risk of complications, with only $1-2 \%$ patients requiring intensive post-operative followup [5]. Routine hospital outpatient follow-up for uncomplicated cataract procedures has been shown not to be necessary, with a very low rate of clinical interventions made at routine post-operative ophthalmology consultations, only $2.8 \%$ [6]. This is in contrast to the $50 \%$ rate of intervention when a patient presents to an eye emergency department with a post-operative issue [6]. Whilst routine follow-up in the ophthalmology clinic is not necessary for most patients after cataract surgery, it is important that a qualified person reviews the outcome of the procedure. Patients may require advice on their post-operative medications and an updated spectacle prescription, and they may wish to discuss options regarding cataract surgery for the second eye. The Royal College of Ophthalmologists Cataract Surgery guidelines outline that a post-operative review can be carried out by an ophthalmologist, nurse, optometrist or orthoptist working within the hospital eye department, or by an accredited optometrist working outside of the hospital [5]. 
Community optometrist-led post-operative care can significantly reduce the workload associated with cataract surgery. It involves suitable patients being discharged on the day of surgery to their local optometrist for their follow-up. Optometrists carrying out the post-operative examination in a shared care pathway prevents duplication of work, as at the post-operative visit with an ophthalmologist the patient is usually advised to attend their optometrist for an updated refraction. Shared care with community optometrists has been shown to be highly effective in cataract care pathways [7, 8]. A previous study carried out by our group showed that community optometrists provide superior post-operative feedback compared to doctors [9]. High sensitivity and specificity for recognition of post-operative complications has been demonstrated in co-managing optometrists [10]. This model of care is additionally rated highly by patients [11]. A shared care pathway is beneficial from a health economics perspective, can assist in reducing outpatient waiting times, and also minimizes unnecessary hospital visits for this elderly cohort, which is particularly important in the era of COVID-19.

It is important to bear in mind that serious complications of cataract surgery such as endophthalmitis and retinal detachment require urgent intervention. It therefore is important that post-operative patients in the community can be easily rereferred to the hospital eye service should a post-operative complication arise. There is paucity of evidence on the proportion of cataract patients in shared care models that must be re-referred to the hospital from the community.

Eight years after the introduction of a shared-care pathway for cataract surgery in Sligo University Hospital, we wished to quantify the number of patients who are discharged to community optometry on the day of surgery, and the proportion that are re-referred or re-present to the hospital eye service due to cataract-related issues.

\section{Methods}

\section{Cataract care pathway}

Our cataract care pathway consists of two pre-operative visits to the hospital eye department, and a four-week post-operative examination with a community optometrist. At the first preoperative appointment the patient is seen by a doctor- a decision is made regarding need and suitability for surgery, and any peri-operative precautions that need to be taken. The doctor can pre-approve whether the patient would be suitable for listing for cataract surgery in their second eye should they wish to be listed post-operatively. The second pre-operative appointment is nurse-led and consists of a medical and anaesthetic history, examination of vital signs, autorefraction and biometry. On the day of cataract surgery, appropriate patients can be discharged to a community optometrist for their post- operative care, at the discretion of the consultant surgeon. An ophthalmic nurse schedules an appointment with a participating optometrist in the patient's locality for a date four weeks post-operatively. Post-operative information is given in a leaflet, including advice on post-operative drop installation, a list of post-operative symptoms for which they should seek urgent attention and an emergency contact number. Those unsuitable for discharge after their cataract surgery (eg. complicated procedure) remain in the hospital ophthalmic service for regular post-operative review. The optometrists are supplied with a standardized form for recording post-operative findings which they can return by post to the hospital ophthalmic department, or alternatively can enter onto the Electronic Patient Record (EPR). The parameters recorded include post-operative visual acuity, refraction and intraocular pressure.

To minimize the risk of post-operative complications, all patients receive intracameral antibiotic during their procedure, unless contraindicated. They are prescribed a combined dexamethasone/neomycin/polymyxin eye drop, and a preservative free lubricating ointment at night time for four weeks post-operatively. Additional post-operative medication may be prescribed as needed at the discretion of the surgeon.

Optometrists participating in our scheme must attend accreditation sessions in the hospital for training in postoperative cataract care and complications, re-referral pathways and Medisoft EPR use. The optometrists in the catchment area have access to all pre-operative and operative notes on the EPR. Consent is explicitly given by the patient to share these clinical records with their optometrist, in line with General Data Protection Regulation. At the post-operative visit, examination findings and refraction can be recorded on the EPR. If the patient wishes to be listed for cataract surgery for their second eye, the optometrist can do this once it was preapproved at their initial pre-operative appointment. If there is concern over a post-operative complication, the ophthalmologist on call can be contacted and the patient seen in eye casualty. The optometrists are paid $€ 44.85$ from the Health Service Executive per patient.

\section{Data collection and analysis}

This is a retrospective review on all patients who underwent cataract surgery in our department between July and October 2019. Theatre registers were used to compile a list of patients who had cataract surgery over the three-month period. Medisoft EPR were used to identify whether these patients were discharged to community optometrists on the day of cataract surgery or kept in the hospital ophthalmic service for their post-operative care. We then identified which patients re-presented or were re-referred to the hospital ophthalmic service for post-operative issues. We recorded all post- 
operative complications. Microsoft Excel was used to obtain descriptive statistics.

\section{Results}

394 cataract procedures were carried out on 379 patients over the three-month period from the beginning of July to the beginning of October 2019. Data was collected from EPR a mean of 204 ( \pm 25 standard deviation) days post-operatively. $63.5 \%$ cataract procedures were carried out by a consultant, $24.9 \%$ were performed by a registrar, $11.4 \%$ by a senior house officer. All patients received antibiotics during their cataract operation- intracameral cefuroxime is routinely given as per local protocol. In four cases, cefuroxime was contraindicatedthese patients received subconjunctival gentamicin instead.

For $369(93.6 \%)$ of the cataract procedures, the patient was discharged to an optometrist for their post-operative care. Post-operative findings were documented on the EPR by the optometrists for $78.6 \%$ of these patients. 3 patients were referred to a medical ophthalmologist whom they attended regularly for their post-operative care, rather than an optometrist. The remaining 22 were kept in the hospital eye service for post-operative review. The reasons for not discharging these patients are shown in Table 1 , and include due to an intraoperative complication, concomitant glaucoma drainage device insertion, or because primary angle closure was the indication for cataract surgery. 3 of the patients who were kept in the hospital eye service for review attended eye casualty outside of their scheduled appointment with a post-operative complication.

38 of the patients who had been discharged to community optometry for post-op care $(10.3 \%)$ were re-referred or represented to the hospital ophthalmic service. $39.5 \%$ of these patients were re-referred by their optometrist, $5.2 \%$ were rereferred by their GP, $23.7 \%$ self-presented, the remainder had an unknown route of referral. $12(31.6 \%)$ of the patients represented prior to their four-week post-operative appointment with the optometrist (see Fig. 1). 7 re-attended within one

Table 1 Reasons for not discharging patients to an optometrist for their post-operative care

\begin{tabular}{ll}
\hline Reason & Number of Patients \\
\hline Intra-operative complication & 5 \\
Insertion of glaucoma drainage device & 4 \\
Pre-existing condition & 4 \\
Primary angle closure glaucoma & 5 \\
Posterior capsulotomy deemed likely to be needed & 1 \\
Surgeons discretion & 3 \\
\hline
\end{tabular}

\section{Timescale of re-presentation}

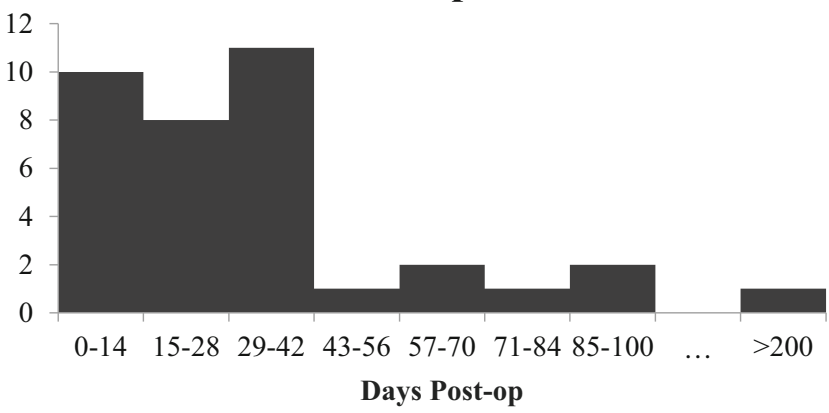

Fig. 1 Number of days post-op that patients re-presented/were re-referred to the hospital ophthalmic department

week of their procedure, 5 re-presented more than two months after their operation.

$21(55 \%)$ of those who re-attended had a post-operative complication. Of the other patients who reattended, 4 were treated for post-operative dry eye, the others had no postoperative issues. Only one case of dry eye was noted to be visually significant. There were no set criteria used for dry eye, and diagnosis was based on symptoms and/or the presence of superficial punctate erosions on the cornea. All patients with dry eye were treated with lubricants.

The total number of post-operative complications was 24 , affecting 24 patients (see Table 2). The most frequent complication was post-operative uveitis which affected 7 patients (1.8\%). All cases of uveitis were mild, with inflammation only in the anterior chamber of the eye. One of the patients was noted to have posterior synechiae, none had a hypopyon. All of the patients with post-operative uveitis were treated successfully using steroid drops as an outpatient. The second most frequent complication was cystoid macular oedema, occurring in 5 patients (1.2\%). $80 \%$ of these patients responded to topical steroids and/or non-steroidal anti-inflammatory drops, one patient required both orbital floor and intra-vitreal steroids for cystoid macular oedema. Four patients had

Table 2 Post-operative complications presenting to the hospital ophthalmic department

\begin{tabular}{ll}
\hline Post-Op Complication & Number of patients \\
\hline Uveitis & 7 \\
Cystoid macular oedema & 5 \\
Corneal oedema & 4 \\
Raised IOP & 3 \\
Retinal detachment & 1 \\
Other & 4 \\
Retained lens fragment & 1 \\
Vitreous haemorrhage & 1 \\
Drop allergy & 1 \\
Conjunctivitis & 1 \\
Total & $\mathbf{2 4}$ \\
\hline
\end{tabular}


corneal oedema, three of which were associated with corneal epithelial dryness. There were three patients with raised intraocular pressure post-operatively. There was one retinal detachment that occurred three days after an uncomplicated procedure in a patient with a background of myopia. Other serious complications included one retained lens fragment. There were no cases of endophthalmitis.

\section{Discussion}

Shared care for uncomplicated post-operative patients is an effective way of reducing the workload associated with cataract surgery. $93.6 \%$ of our patients were deemed suitable for community optometry follow up. $89.7 \%$ of these did not need to be seen again in the hospital ophthalmic department, which corresponds to three hundred and thirty one outpatient appointment slots.

Post-operative data was available on the EPR for $78.6 \%$ patients who were followed up by the community optometrist, meaning that at least this number of patients attended their post-operative appointment. In our previous study, documentation of follow-up on the EPR was present for $83 \%$ of patients. On investigation of the remaining patients, $11 \%$ had attended their appointment but the data was not input onto the EPR by the optometrist. It is therefore likely in our present study that more than our recorded number had attended their post-operative review.

Although our sample size is limited, post-operative complication rates were consistent with those reported in the guidelines [5, 12], which indicates that complications were not being missed. In addition to this, the rate of complications confirm that our local protocol regarding intracameral antibiotics and the postoperative drop regimen is up to standard. There were no cases of endophthalmitis amongst our patients. The intervention rate on patients who presented to eye casualty post-operatively was $55 \%$, similar to that in the study by Allan et al. in 1997 [6]. The patients that did not require intervention perhaps may not have needed to attend.

Dry eye is a relatively common occurrence post cataract surgery [13]. These patients often attend eye casualty with pain or grittiness but can be managed for the most part with over the counter lubricating drops. Dry eye can be managed by community optometrists [14], which could reduce the number of patients re-presenting to the hospital. $75 \%$ of the patients that re-presented to the hospital eye service with dry eye alone attended within the first 10 days post-operatively, prior to their optometrist review. Perhaps these patients could have been managed with over-the-phone advice.

The main limitations of our study was its retrospective nature, as well as variation in terms used by doctors on the EPR when describing their examination findings. Medisoft EPR has a standardized section for recording post-operative complications, however, time in eye casualty may not always permit more than a quick note.

Strong communication links and re-referral pathways from community optometry to the hospital ophthalmic department is imperative for timely management of post-operative complications. Electronic patient records facilitate a smooth transfer of care from the hospital to the community. Our community optometrists can contact our ophthalmologist on call 24-h a day and patients can be seen immediately via eye casualty if there are any concerns. This model of care therefore may be best suited to departments with an eye casualty or rapid access service.

Although this data was collected prior to the COVID-19 pandemic, its results are perhaps more important now. Discharging patients to the community for their postoperative care is a safe, effective and practical approach to minimizing footfall on our hospitals.

Availability of data and material Not applicable.

Authors contributions All authors contributed to the study conception and design. Material preparation, data collection and analysis were performed by Amy O'Regan and Aisling McGlacken-Byrne. This draft of the manuscript was written by Amy O'Regan and Aisling McGlackenByrne, supervised by Shivona Chetty and Paul Mullaney, and all authors commented on previous versions of the manuscript. All authors read and approved the final manuscript.

Funding Not applicable.

\section{Compliance with ethical standards}

Conflicts of interest/competing interests No disclosures.

Code availability Not applicable.

Ethics approval Not applicable.

Consent to participate Not applicable.

Consent for publication Not applicable.

\section{References}

1. G. Lafortune, G. Balestat, and A. Durand, "Directorate for Employment, labour and social affairs comparing activities and performance of the hospital sector in Europe: how many surgical procedures performed as inpatient and day cases?," 2012

2. "Activity in Acute Public Hospitals in Ireland. 2018 Annual Report" HEALTHCARE PRICING OFFICE. 2019

3. "Population Projections Results - CSO - Central Statistics Office." [Online]. Available: https://www.cso.ie/en/releasesandpublications/ep/ p-plfp/populationandlabourforceprojections2017-2051/ populationprojectionsresults/. [Accessed: 16-Dec-2020]

4. LaGanga LR (Jul. 2011) Lean service operations: reflections and new directions for capacity expansion in outpatient clinics. J Oper Manag 29(5):422-433 
5. "The Royal College of Ophthalmologists Cataract Surgery Guidelines," 2010

6. Allan BDS, Baer RM, Heyworth P, Duguid IGM, Dart JKG (Jul. 1997) Conventional routine clinical review may not be necessary after uncomplicated phacoemulsification. Br J Ophthalmol 81(7): $548-550$

7. Voyatzis G, Roberts HW, Keenan J, Rajan MS (2014) Cambridgeshire cataract shared care model: community optometrist-delivered postoperative discharge scheme. Br J Ophthalmol 98(6):760-764

8. D. J. Muthucumarana and T. J. Rimmer, "Cataract surgery and the optometrist," Eye, 2000

9. Mongan AM, Kerins F, McKenna B, Quinn SM, Mullaney P (May 2018) Changing trends in postoperative cataract care: impact of electronic patient records in optometrist-delivered shared care. Ir J Med Sci 187(2):529-535

10. Revicki DA, Poe ML (1995) Quality of care in cataract surgery cases experiencing post-operative complications with co-managed care. J. Am. Optom. Assoc
11. Van Vliet EJ, Reus NJ, Sermeus W, Vissers JMH, Sol JCA, Lemij HG (Oct. 2010) Patients' experiences and preferences with comanaged care in a cataract pathway. Br J Ophthalmol 94(10): $1363-1368$

12. R. J. Olson et al., "Cataract in the Adult Eye Preferred Practice Pattern®," Ophthalmology, vol. 124, no. 2, pp. P1-P119, 2017

13. Sutu C, Fukuoka H, Afshari NA (Jan. 2016) Mechanisms and management of dry eye in cataract surgery patients. Curr Opin Ophthalmol 27(1):24-30

14. Needle JJ, Petchey R, Lawrenson JG (2008) A survey of the scope of therapeutic practice by UK optometrists and their attitudes to an extended prescribing role. In: A survey of the scope of therapeutic practice by UK optometrists and their attitudes to an extended prescribing role. Ophthalmic Physiol. Opt

Publisher's note Springer Nature remains neutral with regard to jurisdictional claims in published maps and institutional affiliations. 